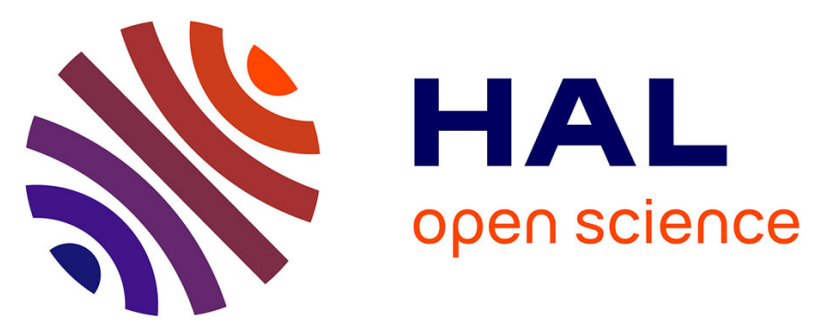

\title{
Extended braking stiffness estimation based on a switched observer, with an application to wheel-acceleration control
}

Trong-Bien Hoang, William Pasillas-Lépine, Alexandre de Bernardinis, Mariana Netto

\section{To cite this version:}

Trong-Bien Hoang, William Pasillas-Lépine, Alexandre de Bernardinis, Mariana Netto. Extended braking stiffness estimation based on a switched observer, with an application to wheelacceleration control. IEEE Transactions on Control Systems Technology, 2014, 22 (6), pp.2384-2392. 10.1109/TCST.2014.2305171 . hal-01104886

\section{HAL Id: hal-01104886 \\ https://hal-centralesupelec.archives-ouvertes.fr/hal-01104886}

Submitted on 19 Jan 2015

HAL is a multi-disciplinary open access archive for the deposit and dissemination of scientific research documents, whether they are published or not. The documents may come from teaching and research institutions in France or abroad, or from public or private research centers.
L'archive ouverte pluridisciplinaire HAL, est destinée au dépôt et à la diffusion de documents scientifiques de niveau recherche, publiés ou non, émanant des établissements d'enseignement et de recherche français ou étrangers, des laboratoires publics ou privés. 


\title{
Extended braking stiffness estimation based on a switched observer, with an application to wheel-acceleration control ${ }^{*}$
}

\author{
Trọng Biên Hoàng ${ }^{1}$, William Pasillas-Lépine ${ }^{1}$, Alexandre De Bernardinis ${ }^{2}$ and Mariana Netto ${ }^{3}$
}

\begin{abstract}
In the context of hybrid anti-lock brake systems (ABS), a closed-loop wheel-acceleration controller based on the observation of the extended braking stiffness (XBS) is provided. Its objective is to improve the system's robustness with respect to changes in the environment (as changes in road conditions, brake properties, etc.). The observer design is based on Burckhardt's tyre model, which provides a wheel acceleration dynamics that is linear up to time-scaling. The XBS is one of the state variables of this model. The paper's main result is an observer that estimates this unmeasured variable. When the road conditions are known, a three-dimensional observer solves the problem. But, for unknown road conditions, a more complex four-dimensional observer must be used instead. In both cases, the observer's convergence is analysed using tools for switched linear systems that ensure uniform exponential stability (provided that a dwelltime condition is satisfied). Both experiments and simulations confirm the convergence properties predicted by the theoretical analysis.
\end{abstract}

Index Terms-Automotive control, Anti-lock brake systems, Observer design, Switched linear systems, Lyapunov stability.

\section{INTRODUCTION}

The anti-lock brake system (ABS) is now a standard equipment on most new passenger cars, in order to prevent wheel lock-up and limit the risk of skidding. With this system, the car maintains its steerability and reduces its braking distance, even in the case of an emergency braking. Historically, the first commercial ABS systems were designed using logicbased switching controllers, in which the mode changes are determined by the evolution of the wheel's angular acceleration (see, e.g., [20], [19], [18], [4]). The main force of these controllers is that they avoid the use of the (unmeasured) wheel slip and of its (unknown) optimal value. They are therefore quite robust with respect to changes in tyre parameters and road conditions. Their main drawback is, however, that they were derived from purely heuristic arguments and are, as a consequence, difficult to tune. Despite of this, the ABS controllers present on today's commercial vehicles mainly belong to this category. More recently, mainly in an academic context, several wheel slip controllers have been proposed in

\footnotetext{
* The research leading to these results has been supported by the French region Ile-de-France, through the Regeneo project (RTRA Digiteo and DIM LSC). It has also received funding from the European Union Seventh Framework Programme [FP7/2007-2013] under grant agreement no 257462 HYCON2 Network of excellence.

${ }^{1}$ T. B. Hoàng (bien.hoangelss.supelec.fr) is with L2SUniversité Paris Sud-Supélec, 3 rue Joliot-Curie, 91192 Gif-sur-Yvette, France. And W. Pasillas-Lépine (pasillas@lss.supelec.fr) is with CNRS-L2S-Supélec (same address).

${ }^{2}$ A. De Bernardinis (alexandre.de-bernardiniseifsttar.fr) is with IFSTTAR COSYS-LTN, 25 allée des Marronniers, 78000 Versailles, France.

${ }^{3} \mathrm{M}$. Netto (mariana. nettodifsttar. $\mathrm{fr}$ ) is with IFSTTAR COSYSLIVIC, 14 route de la Minière, Bât. 824, 78000 Versailles, France.
}

the literature (see, e.g., [34], [17], [30], [7], [28]). The main interest of these controllers is that they apply a brake torque that converges to a specific value, which avoids the typical limit cycles generated by logic-switched algorithms. This leads to shorter braking distances, at least on standard road conditions. Unfortunately, these approaches assume (implicitly) that the wheel slip is measured (or estimated) and that its optimal value is known, two requirements that are often difficult to meet. Even if such algorithms might not be robust enough to be implemented on commercial ABS, they are still useful for some specific applications [35], like the electronic stability program (ESP).

In addition to hybrid and continuous approaches for ABS, which both have their pros and cons [31], one can find a different research line (see, e.g., [12], [25], [32]) based on the concept of extended braking stiffness (XBS). The XBS is the slope $\mu^{\prime}(\lambda)$ of the tyre characteristic $\mu(\lambda)$. For additional details, the reader is referred to Section II. In standard conditions, there exists an (unknown) value of the wheel slip $\lambda^{*}$ for which the curve $\mu$ reaches its maximum. That is, such that $\mu^{\prime}\left(\lambda^{*}\right)=0$. The main theoretical interest of XBS for braking strategies is hence clear: unlike wheel slip, that has an unknown optimal target value $\lambda^{*}$, the optimal value of XBS is always the same (zero). An intuitive approach for ABS control is thus to regulate the value of XBS around zero. But, actually, the XBS appears also in other contexts related to braking systems. A first example is wheel acceleration control. In this context, the XBS can be seen as a disturbance that must be compensated in order to increase either the controller's bandwidth or its delay margin (see, e.g., [8], [11], [15]). A second (related) example is wheel slip control. Indeed, since the wheel acceleration is closely related to the derivative of the wheel slip (see Section II-B), the XBS appears also naturally in this domain [28]. One should stress, however, that the XBS cannot be measured directly using standard sensors. In order to use it in a control algorithm one must therefore address first its real-time estimation, which is the main objective of this paper. Because of the diversity of control problems in which the XBS appears, it would have been difficult to treat all of them here. The choice of the authors was thus to emphasize the contributions associated to the estimation problem, and to consider the control issues only for illustration purposes.

The simplest approach to estimate $\mathrm{XBS}$ is probably to consider this variable as a constant parameter, which allows the use of online least squares methods [25]. Other approaches analyze the tyre/carcass resonance in the frequency domain [32] or use algebraic methods [36]. Solutions based on wheel slip measurements are also available [33]. Nevertheless, to the author's knowledge, the idea of exploiting the nonlinear XBS dynamics in a model-based observer has not been considered 
before in the literature, at least in the case of the longitudinal stiffness. The approach proposed in this paper is based on a new model for the wheel acceleration dynamics. In this model, the extended braking stiffness enters as one of the state variables. When the road conditions are known this model is three-dimensional. Otherwise a fourth order dynamics is obtained. In both cases an observer can be constructed using a copy of the system's dynamics and adding a nonlinear correction term that is proportional to the observation error. After a suitable time-rescaling, the observer error is reduced to a linear switched system that can be analyzed using standard methods [13]. When the observer switches admit a strictly positive dwell-time, the observer's convergence is global, uniform, and exponential. Compared to previous works, the authors believe that the main interest of this method comes from its simplicity and from the fact that the parameters of the tyre model are not needed by the proposed algorithm.

In order to illustrate on a concrete example the interest of this observer, the case of a simple academic ABS strategy [27] is considered. In their standard form, this kind of algorithms might fail to cycle correctly [1] when there are significant changes in the environment (as changes in road conditions, brake properties, etc.). In a recent work [11], it has been shown that adding closed-loop wheel acceleration control during the phases for which the brake pressure is modified can compensate this lack of robustness. But, in order to reach the bandwidth required by this kind of controllers, an XBS estimate is necessary. The combination of such control laws with the proposed XBS observer has been tested both on simulations (with changes of road conditions) and experimentally (with constant road conditions, imposed by the test-rig characteristics).

This paper is organized as follows. First, the system's dynamics is described in Section 2. Then, the main contributions of the paper (the design and the stability analysis of two switched observers) are presented in Sections 3 and 4, with the corresponding experimental and simulation results. An academic five-phase hybrid ABS and a closed-loop wheelacceleration control law are briefly described in Section 5, in order to exhibit a potential application for these observers. Finally, concluding remarks and perspectives for future research are presented in Section 6.

\section{SySTEM MODELLING}

The basic dynamics of the wheel, which is central to this study, can be analyzed using a single-wheel model (see, e.g., [24] and [25]). The main reason for using this model is that, despite of its simplicity, all the basic phenomena related to ABS control appear in it [11].

\section{A. Wheel dynamics}

The angular velocity $\omega$ of the wheel has the following dynamics:

$$
I \frac{d \omega}{d t}=-R F_{x}+T_{w}
$$

where $I$ denotes the inertia of the wheel, $R$ its radius, $F_{x}$ the longitudinal tyre force, and $T_{w}$ the torque applied to the

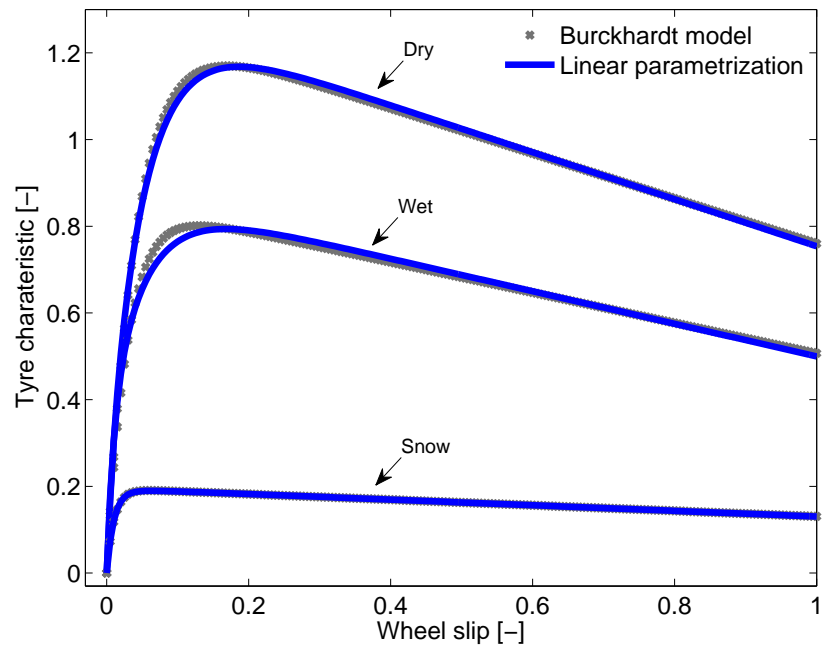

Fig. 1. Comparison of the tyre characteristic $\mu(\lambda)$ given by Burckhardt's model (5) and its approximation (18), on different road conditions. For clarity, only the positive wheel slip part of the curve is shown (instead of the negative part, which corresponds to braking). The parameters of the tyre models are given in Table I (Section III).

wheel. The torque $T_{w}=T_{e}-T_{b}$ is composed of the engine torque $T_{e}$ and the brake torque $T_{b}$. It is assumed that during ABS braking the clutch is open and thus the engine torque is neglected. In other words, $T_{b}=\gamma_{b} P_{b}$, where $P_{b}>0$ denotes the brake pressure and $\gamma_{b}>0$ the brake efficiency.

The longitudinal tyre force $F_{x}$ is often modelled by the relation

$$
F_{x}=\mu(\lambda) F_{z}
$$

where $F_{z}$ denotes the vertical load and

$$
\lambda=\frac{R \omega-v_{x}}{v_{x}}
$$

denotes the wheel slip [18]. The longitudinal speed of the vehicle $v_{x}$, which is considered as an external variable of the model, is assumed to be strictly positive. In a braking manoeuvre, this implies $\lambda<0$ and $F_{x}<0$. The tyre characteristic $\mu(\cdot)$ is a function that is both smooth and odd. It satisfies $\mu(0)=0$ and $\mu^{\prime}(0)>0$ (see Figure 1), where $\mu^{\prime}(\lambda)$ denotes the derivative of $\mu$ with respect to $\lambda$. Several mathematical descriptions are available in order to describe this curve. Two of them are considered in Sections III-A and IV-A.

\section{B. Wheel acceleration dynamics}

The state variables of the model are

$$
x_{1}=\lambda \quad \text { and } \quad x_{2}=R \frac{d \omega}{d t}-a_{x}(t),
$$

where $a_{x}(t)=d v_{x}(t) / d t$ denotes the vehicle's longitudinal acceleration. The state $x_{1}$ is the wheel slip. The state $x_{2}$ is the wheel acceleration offset, that is, the difference between the acceleration of the wheel and that of the vehicle. These 
variables evolve with the following dynamics

$$
\begin{aligned}
\frac{d x_{1}}{d t} & =\frac{1}{v_{x}(t)}\left(-a_{x}(t) x_{1}+x_{2}\right) \\
\frac{d x_{2}}{d t} & =-\frac{a \mu^{\prime}\left(x_{1}\right)}{v_{x}(t)}\left(-a_{x}(t) x_{1}+x_{2}\right)+\frac{R}{I} \frac{d T_{w}}{d t}-\frac{d a_{x}(t)}{d t},
\end{aligned}
$$

where $a=\left(R^{2} / I\right) F_{z}$ and the extended braking stiffness $\mu^{\prime}($. is defined as the derivative of the tyre characteristic $\mu($.$) with$ respect to $\lambda$.

During an ABS-controlled braking manoeuvre, the vehicle's acceleration $a_{x}(t)$ stays almost constant and close to the maximal value $a_{x}^{*}$ allowed by the road's conditions [11]. Moreover, the wheel slip $\lambda$ remains relatively small. In such conditions, the control and observer designs can be simplified by considering that $\left(-a_{x} x_{1}+x_{2}\right) \simeq x_{2}$. This approximation is exact only at constant speed, but it remains reasonable in the case of ABS manoeuvres [11]. Its validity is checked $a$ posteriori in Sections III and IV, by simulating the proposed observers on the original (non-simplified) model. This approximation leads to a simpler dynamics

$$
\begin{aligned}
& \frac{d x_{1}}{d t}=\frac{1}{v_{x}(t)} x_{2} \\
& \frac{d x_{2}}{d t}=-\frac{a}{v_{x}(t)} \mu^{\prime}\left(x_{1}\right) x_{2}+b u,
\end{aligned}
$$

where the control variable $u=d P_{b}(t) / d t$ is the derivative of the brake pressure and $b=-R \gamma_{b} / I$. Indeed, we have $T_{w}=$ $-\gamma_{b} P_{b}$ (see Section II-A), and thus $(R / I) d T_{w} / d t=b d P_{b} / d t$.

\section{OBSERVER DESIGN (KNOWN ROAD CONDITIONS)}

\section{A. Tyre characteristic}

In the literature, one can find several mathematical formulas that have been used to describe the tyre characteristic $\mu(\lambda)$, such as trigonometric functions in [26], second order rational fractions in [18] and [27], and exponentials in [5]. This section is based on Burckhardt's [5] model

$$
\mu(\lambda)=c_{1}\left(1-e^{-c_{2} \lambda}\right)-c_{3} \lambda,
$$

where the coefficients $c_{i}$ are constants depending on the road conditions, the tyre model, the tyre pressure, etc. Therefore, for the sake of robustness, the ABS algorithms should be able to handle the uncertainty associated with these coefficients. A typical tyre characteristic associated to this model is illustrated in Figure 1.

\section{B. Extended braking stiffness dynamics}

Burckhardt's tyre model is particularly interesting when it comes to estimate the value of the extended braking stiffness, which cannot be measured directly. Indeed, a simple mathematical formula for $\mu^{\prime}(\lambda)$ can be obtained by differentiating (5), with respect to $\lambda$. From this formula and from the second order derivative of (5), one can establish a relation between these variables:

$$
\mu^{\prime \prime}(\lambda)+c_{2} \mu^{\prime}(\lambda)+c_{2} c_{3}=0
$$

Now, defining the wheel acceleration offset $z_{1}=x_{2}$, the extended braking stiffness $z_{2}=\mu^{\prime}\left(x_{1}\right)$, and the unknown

\begin{tabular}{|c||c|c|c||c|c|c|}
\hline \multicolumn{1}{|c||}{} & \multicolumn{2}{c||}{ Burckhardt's model } & \multicolumn{3}{c|}{ Approximate model } \\
\cline { 2 - 7 } & $c_{1}$ & $c_{2}$ & $c_{3}$ & $\theta_{0}$ & $\theta_{1}$ & $\theta_{2}$ \\
\hline Dry road & 1.28 & 24 & 0.52 & -0.53 & 25.22 & 7.2 \\
\hline Wet road & 0.86 & 34 & 0.35 & -0.36 & 8.86 & 24 \\
\hline Snow & 0.28 & 50 & 0.05 & -0.05 & 0.24 & 14 \\
\hline
\end{tabular}

Table I - Tyre parameters for Burckhardt's model.

product of parameters $z_{3}=-c_{2} c_{3}$ as new variables, combining equations (4) and (6) gives

$$
\begin{aligned}
\frac{d z_{1}}{d t} & =\frac{-a}{v_{x}(t)} z_{1} z_{2}+b u \\
\frac{d z_{2}}{d t} & =\left(c z_{2}+z_{3}\right) \frac{z_{1}}{v_{x}(t)} \\
\frac{d z_{3}}{d t} & =0
\end{aligned}
$$

where $c=-c_{2}$ is a constant that depends on road conditions. This model can be seen as a generalization of the model proposed in [25] and as a particular case of (4), associated to Burckhardt's tyre model. Somehow, considering the unknown constant $z_{3}$ as a new state variable (and not as a parameter) is not optimal. Indeed, the adaptive observer approach [3] could have been a more standard way to handle this problem. Nevertheless, that approach has not been followed here because (for the authors) it is not obvious how to combine it with the switchings introduced in the next section. While, using a representation of the form (7), the approach of [16] is directly applicable.

On the one hand, an interesting quality of this model is that the wheel slip (which cannot be measured) does not appear explicitly in it as a state variable. One might argue that this is not that interesting, since the velocity (which cannot be measured neither) appears instead in the system's dynamics. Nevertheless, at least at high speeds, it is much easier to estimate the vehicle's velocity than to estimate wheel slip [10]. On the other hand, the main drawback of our model (7) is that it is assumed that the value of $c$ is known, which is true only for a fixed type of road conditions (the more complex case of unknown road conditions is considered later, in Section IV).

\section{Observer Design}

Since, unlike the wheel acceleration offset $z_{1}$, the extended braking stiffness $z_{2}$ is not directly measurable, it must be estimated using an observer. To that aim, one can start with a copy of the original system and add some terms proportional to the observation error, in order to ensure the convergence of the trajectories between both systems. As it is shown later, multiplying these terms by $z_{1}$ simplifies considerably the analysis. At the end, one obtains

$$
\begin{aligned}
\frac{d \widehat{z}_{1}}{d t} & =\frac{-a}{v_{x}} z_{1} \widehat{z}_{2}+b u+\frac{k_{1}\left(z_{1}\right)}{v_{x}} z_{1}\left(z_{1}-\widehat{z}_{1}\right) \\
\frac{d \widehat{z}_{2}}{d t} & =\left(c \widehat{z}_{2}+\widehat{z}_{3}\right) \frac{z_{1}}{v_{x}}+\frac{k_{2}\left(z_{1}\right)}{v_{x}} z_{1}\left(z_{1}-\widehat{z}_{1}\right) \\
\frac{d \widehat{z}_{3}}{d t} & =\frac{k_{3}\left(z_{1}\right)}{v_{x}} z_{1}\left(z_{1}-\widehat{z}_{1}\right),
\end{aligned}
$$

where $\widehat{z}_{i}$ are the observer states. 
In (8), the observer gains $k_{i}\left(z_{1}\right)$, for $1 \leq i \leq 3$, must depend on the value of $z_{1}$ in order to ensure the observer's stability independently of the sign of $z_{1}$. The simplest choice might be

$$
k_{i}\left(z_{1}\right)= \begin{cases}k_{i}^{+} & \text {if } z_{1}>0 \\ k_{i}^{-} & \text {if } z_{1}<0 .\end{cases}
$$

Even if the gains $k_{i}\left(z_{1}\right)$ are discontinuous, it must be stressed that the observer gains $k_{i}\left(z_{1}\right) z_{i}$ are continuous, which ensures the existence and uniqueness of solutions for (8) when $z_{1}(t)$ is considered as an external input.

Consider the observer errors $e_{i}:=z_{i}-\widehat{z}_{i}$, for $1 \leq i \leq 3$. Subtracting equation (8) from equation (7) leads to

$$
\frac{d e}{d t}=\frac{z_{1}}{v_{x}}\left(\begin{array}{ccc}
-k_{1}\left(z_{1}\right) & -a & 0 \\
-k_{2}\left(z_{1}\right) & c & 1 \\
-k_{3}\left(z_{1}\right) & 0 & 0
\end{array}\right) e .
$$

Notice that if the right hand side of (10) is divided by $z_{1} / v_{x}$ then the observer error dynamics is transformed into a linear system. This leads to the idea of changing the time-scaling. Indeed, let

$$
s(t):=\int_{0}^{t} \frac{\left|z_{1}(\tau)\right|}{v_{x}(\tau)} d \tau,
$$

which ensures that $d t / d s>0$, independently of the values of $z_{1}$. Since, for any function $\varphi: \mathbb{R} \rightarrow \mathbb{R}^{n}$, one has

$$
\frac{d \varphi}{d s}=\frac{d \varphi}{d t} \frac{d t}{d s}=\frac{d \varphi}{d t} \frac{v_{x}}{\left|z_{1}(t)\right|} .
$$

This implies

$$
\frac{d e}{d s}=\left\{\begin{array}{c}
A_{+} e=\left(\begin{array}{ccc}
-k_{1}^{+} & -a & 0 \\
-k_{2}^{+} & c & 1 \\
-k_{3}^{+} & 0 & 0
\end{array}\right) e \quad \text { if } z_{1}>0 \\
A_{-} e=\left(\begin{array}{ccc}
k_{1}^{-} & a & 0 \\
k_{2}^{-} & -c & -1 \\
k_{3}^{-} & 0 & 0
\end{array}\right) e \quad \text { if } z_{1}<0
\end{array}\right.
$$

which can be written using a more compact notation in the form

$$
\frac{d e(s)}{d s}=A_{\sigma(s)} e(s),
$$

where $\sigma$ denotes a piecewise constant signal that selects, at each instant, a matrix from the pair $\left\{A_{+}, A_{-}\right\}$.

\section{Stability Conditions}

It results from the previous section that the analysis of the asymptotic convergence of the observer (8) can be derived from the stability analysis of the error equation (14), which is an autonomous switched linear system. It appears that numerous stability results are available for that class of systems [21]. Most of them are based on classical Lyapunov-functions. But some LaSalle-like results are also available [13], for which the stability properties of the switched system are proved via regularity assumptions on the set of switching signals.

Define the switching signal $\sigma(t)=\operatorname{sign}\left(z_{1}(t)\right)$, and assume that the solutions of (14) are such that $e$ and $\sigma$ are piecewise differentiable and piecewise constant, respectively. Following [22], define moreover the set $\mathcal{S}\left[\tau_{D}\right]$, with $\tau_{D}>0$, of switchings for which any two consecutive discontinuities of $\sigma$ are separated by no less than $\tau_{D}$. The constant $\tau_{D}$ is called the dwell-time. The origin of a switched system of the form (14) is said to be uniformly exponentially stable if there exists constants $c_{0}$ and $\lambda_{0}$ such that, for each $t \geq 0$, we have $\|e(t)\| \leq c_{0} \exp \left(-\lambda_{0} t\right)\|e(0)\|$. In this definition, the word uniform refers to the fact that $c_{0}$ and $\lambda_{0}$ do not depend on the switching signal [2].

Under a dwell-time condition, as a particular case of Theorem 4 of [13], one can prove that a switched linear system is uniformly exponentially stable if there exists a symmetric positive definite matrix that satisfies simultaneously two nonstrict Lyapunov equations (more details on this point are given in the Appendix). The aim of Theorem 1 below is to show that, for the switched system (14), it is always possible to find a pair of gains $K^{+}$and $K^{-}$such that this LaSalle-like condition is satisfied. To ensure the stability of (14), a first natural condition is to impose the matrices $A_{+}$and $A_{-}$to be Hurwitz. The corresponding conditions on the observer gains can be derived using Routh's criterion, which gives

$$
\begin{aligned}
k_{1}^{+}>c, & k_{2}^{+}<-\frac{c}{a} k_{1}^{+}, \quad \text { and } \\
- & \frac{\left(c k_{1}^{+}+a k_{2}^{+}\right)\left(c-k_{1}^{+}\right)}{a}<k_{3}^{+}<0 ;
\end{aligned}
$$

and

$$
\begin{aligned}
k_{1}^{-}<c, \quad k_{2}^{-}<-\frac{c}{a} k_{1}^{-}, \quad \text { and } \\
0<k_{3}^{-}<-\frac{\left(c k_{1}^{-}+a k_{2}^{-}\right)\left(c-k_{1}^{-}\right)}{a} .
\end{aligned}
$$

From these conditions, with the help of Theorem 4 of [13], one can obtain the following result (proved in the Appendix, at the end of the paper).

Theorem 1 Assume that the three following conditions are satisfied

(i) The gain $K^{+}=\left(\begin{array}{lll}k_{1}^{+} & k_{2}^{+} & k_{3}^{+}\end{array}\right)$satisfies (15).

(ii) The gain $K^{-}=\left(\begin{array}{lll}k_{1}^{-} & k_{2}^{-} & k_{3}^{-}\end{array}\right)$satisfies (16).

(iii) The gains $K^{+}$and $K^{-}$satisfy

$$
\begin{aligned}
\frac{\left(c-k_{1}^{-}\right)}{a k_{3}^{-}}=\frac{\left(c-k_{1}^{+}\right)}{a k_{3}^{+}}>0 \text { and } \\
\left(c k_{1}^{+}+a k_{2}^{+}\right)=\left(c k_{1}^{-}+a k_{2}^{-}\right)<0 .
\end{aligned}
$$

Then, the system (14) is uniformly exponentially stable, provided that the switching signal $\sigma$ admits a strictly positive dwell-time.

This result gives at least a certain degree of freedom: we can chose any $K^{+}$that stabilizes the system. Once this choice has been made, it imposes however an almost unique choice for $K^{-}$(in order to assign the same spectrum to $A_{+}$and $A_{-}$). We do not know, in general, if this constraint can be avoided, but this issue is discussed in [16].

\section{E. Experimental Results}

The observer design proposed in this section has been validated on data coming from the tyre-in-the-loop setup of 

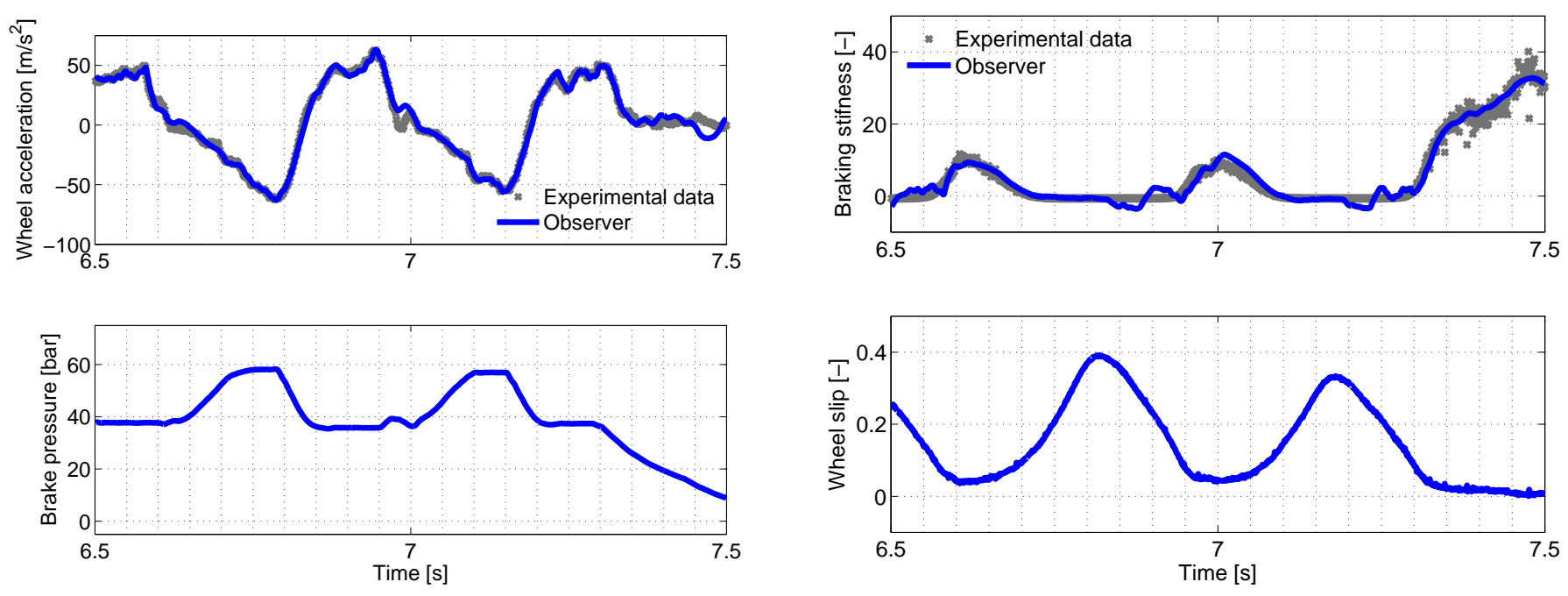

Fig. 2. Comparison between experimental measurements and the estimated states given by the observer (8), during an ABS test [27]. The parameters of the test-rig's tyre characteristics are: $c_{1}=-1.24, c_{2}=-34$, and $c_{3}=0.65$. The system's parameters are: $I=1.2 \mathrm{~kg} \cdot \mathrm{m}^{2}, R=0.3 \mathrm{~m}, F_{z}=2500 \mathrm{~N}$, and $\gamma_{b}=17.5 \mathrm{~N} . \mathrm{m} / \mathrm{bar}$. The speed of the drum is $65 \mathrm{~km} / \mathrm{h}$. The XBS estimated by the observer is compared to the theoretical value obtained from the wheel slip and the derivative of the tyre characteristic.

TU Delft, acquired in the context of ABS research [11]. The test-rig consists in a large steel drum on top of which the tyre is rolling. The tyre is mounted on a wheel that is attached to a rotating axle, which has a rigidly constrained height. The axle is supported by two bearings on both sides of the wheel. The bearing housings are connected to a fixed frame by means of piezo-electric force transducers. A hydraulic disk brake is mounted on one side of the axle. The pressure in the calliper is locally controlled by an analog electronic circuit connected to a servo-valve, in order to match the reference pressure. An illustration of this test-rig can be seen on Section 9.4 of [26]. The setup has been used for several years, at TU Delft, for tyre modelling and identification (see [26, Section 9.4] and [37, Appendix A], and the references therein).

In order to satisfy the conditions imposed by Theorem 1, for positive $z_{1}$ 's, the following observer gains are chosen

$$
\begin{aligned}
k_{1}^{+} & =c+\left(\beta_{1}+2 \beta_{2}\right) \\
k_{2}^{+} & =-\left(\beta_{2}^{2}+2 \beta_{1} \beta_{2}+c k_{1}^{+}\right) / a \\
k_{3}^{+} & =-\beta_{1} \beta_{2}^{2} / a .
\end{aligned}
$$

And, for negative $z_{1}$ 's,

$$
\begin{aligned}
& k_{1}^{-}=c-\left(\beta_{1}+2 \beta_{2}\right) \\
& k_{2}^{-}=-\left(\beta_{2}^{2}+2 \beta_{1} \beta_{2}+c k_{1}^{-}\right) / a \\
& k_{3}^{-}=\beta_{1} \beta_{2}^{2} / a,
\end{aligned}
$$

where $\beta_{1}$ and $\beta_{2}$ are positive constants that assign the spectrum of the error's dynamics. More precisely, the error dynamics (13) will always have two real eigenvalues $-\beta_{1}$ (with multiplicity 1 ) and $-\beta_{2}$ (with multiplicity 2 ), independently of the sign of $z_{1}$. The interest of assigning the same spectrum to $A_{+}$and $A_{-}$is explained in [16].

The experimental results are shown on Figure 2, where it can be seen that the states of the system and of the observer remain close to each other. In this figure, the observer's variable $\hat{z}_{1}$ is compared to the measure of $z_{1}$, while the variable $\hat{z}_{2}$ is compared to an estimation $\mu^{\prime}\left(x_{1}\right)$ of the XBS obtained directly from the measure of wheel slip. One can observe in this figure a surprising phenomenon: the noise of the observed variable $\hat{z}_{2}$ is bigger when the wheel acceleration is positive. A possible explanation for these oscillations might be that the norm of $K^{+}$is bigger than that of $K^{-}$, a constraint imposed by Theorem 1 .

This phenomenon reduces the accuracy of the estimation, which is nevertheless good enough to detect whether the tyre is in its stable or unstable region. The proposed observer has however another weak point: it only works correctly when the parameter $c_{2}$ of Burckhardt's model is known, at least approximatively. The knowledge of this parameter is closely related to the knowledge of road conditions, a problem that is considered in the next section.

\section{OBSERVER DESIGN (UNKNOWN ROAD CONDITIONS)}

In contrast to the simpler approach of Section III, it is now assumed that the observer does not have any information on the road conditions (and thus on the parameters of Burckhardt's model). This new context imposes the use a more complex four-dimensional observer, which can be considered as a generalization of the previous three-dimensional observer (8).

\section{A. Tyre characteristic}

The main difficulty with Burckhardt's model (5) is that its parametrization is nonlinear. Recently, in [33], an alternative parametrization of this model by exponentials has been proposed (see also [6]). This kind of approximations can be traced back up to the work of Prony [29] (see [14] for a modern treatment). In this section, Burckhardt's model is approximated with a similar parametrization

$$
\mu(\lambda)=\theta_{0} \lambda+\theta_{1} \frac{e^{d_{1} \lambda}-1}{d_{1}}+\theta_{2} \frac{e^{d_{2} \lambda}-1}{d_{2}}
$$



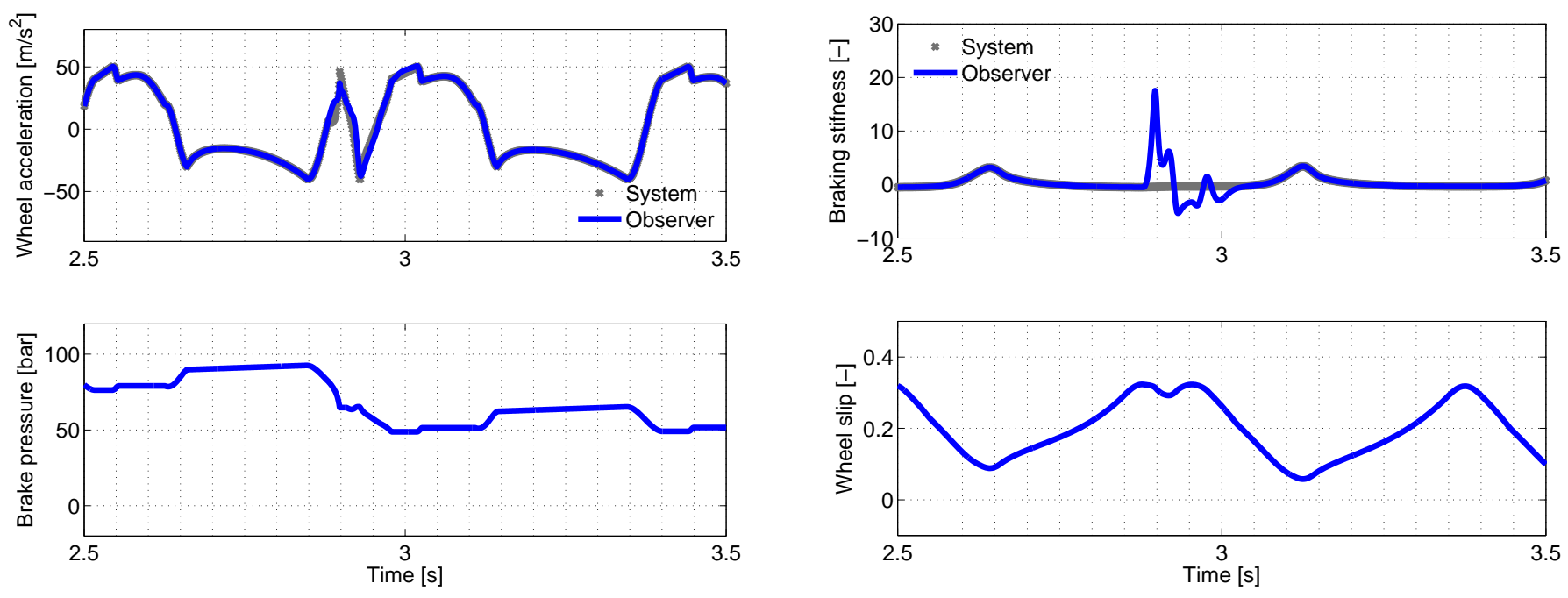

Fig. 3. Simulation of a braking ABS scenario with changes of road conditions: The car runs on dry asphalt during three seconds and on wet asphalt afterwards. In this figure, it can be seen that the XBS observer is highly perturbed by the swift road transition but that, once the transition is over, it converges again in a fraction of a second towards the appropriate state.

defined for $\lambda \leq 0$. The constants $d_{1}$ and $d_{2}$ must be chosen in such a way that $-c_{2} \in\left[d_{1}, d_{2}\right]$. Since, for negative wheel slip, the parameter $c_{2}$ varies in the range $[-50,-24]$, one can take $d_{1}=22$ and $d_{2}=52$. The parameters of Burckhardt's model are shown in Table I. For different road conditions, the coefficients $\theta_{i}$ can be identified using the Least Squares method (see Table I). In Figure 1, the tyre characteristics given by Burckhardt's model (5) is compared to its approximation (18).

\section{B. Extended braking stiffness dynamics}

Computing the first, second and third derivatives of the approximate model (18), with respect to $\lambda$, one can see that these derivatives satisfy the following relation

$$
\mu^{\prime \prime \prime}(\lambda)=\alpha_{0}+\alpha_{1} \mu^{\prime}(\lambda)+\alpha_{2} \mu^{\prime \prime}(\lambda),
$$

where $\alpha_{0}=d_{1} d_{2} \theta_{0}, \alpha_{1}=-d_{1} d_{2}, \alpha_{2}=\left(d_{1}+d_{2}\right)$. Therefore, following the ideas of Section III, we take as state variables $z_{1}=x_{2}, z_{2}=\mu^{\prime}(\lambda), z_{3}=\mu^{\prime \prime}(\lambda)$, and $z_{4}=\alpha_{0}$. Now, combining equations (4) and (19) gives

$$
\begin{aligned}
\frac{d z_{1}}{d t} & =\frac{-a}{v_{x}(t)} z_{1} z_{2}+b u(t) \\
\frac{d z_{2}}{d t} & =\frac{z_{1}}{v_{x}(t)} z_{3} \\
\frac{d z_{3}}{d t} & =\frac{z_{1}}{v_{x}(t)}\left(\alpha_{1} z_{2}+\alpha_{2} z_{3}+z_{4}\right) \\
\frac{d z_{4}}{d t} & =0
\end{aligned}
$$

where $a$ and $b$ are defined in Section II.

The most important property of this model is that the parameters $\alpha_{1}$ and $\alpha_{2}$ do not depend on road conditions. This leads to the possibility of observing the extended braking stiffness, using neither the wheel slip nor the parameters that describe the tyre characteristic. It should be stressed, however, that this model is only valid for constant road conditions. In the case of a change of road conditions (see, e.g., Figure 3), the validity of the model fails temporarily, which might induce a brief divergence between the system's and the observer states.

\section{Observer design}

For system (20), an observer with an error dynamics that is linearizable by a time-scaling can be constructed following the same approach as in Section III. This leads to a switching error dynamics (13), with

$$
A_{+}=\left(\begin{array}{cccc}
-k_{1}^{+} & -a & 0 & 0 \\
-k_{2}^{+} & 0 & 1 & 0 \\
-k_{3}^{+} & \alpha_{1} & \alpha_{2} & 1 \\
-k_{4}^{+} & 0 & 0 & 0
\end{array}\right)
$$

and

$$
A_{-}=\left(\begin{array}{cccc}
k_{1}^{-} & a & 0 & 0 \\
k_{2}^{-} & 0 & -1 & 0 \\
k_{3}^{-} & -\alpha_{1} & -\alpha_{2} & -1 \\
k_{4}^{-} & 0 & 0 & 0
\end{array}\right) .
$$

Conditions for the stability of (14), in the case of these new matrices $A_{+}$and $A_{-}$, can be derived following the same approach as for Theorem 1 (see the Appendix).

\section{Simulation results}

In test-rigs like those of TU Delft, changes of road conditions are not possible. Nevertheless, numerical simulations can still be used to assess the performance of the proposed observer. This has been done considering the (non-simplified) model of Section II and using the observer's output to implement the control law of Section V. In order to ensure the observer's stability, for positive $z_{1}$ 's, the following observer 

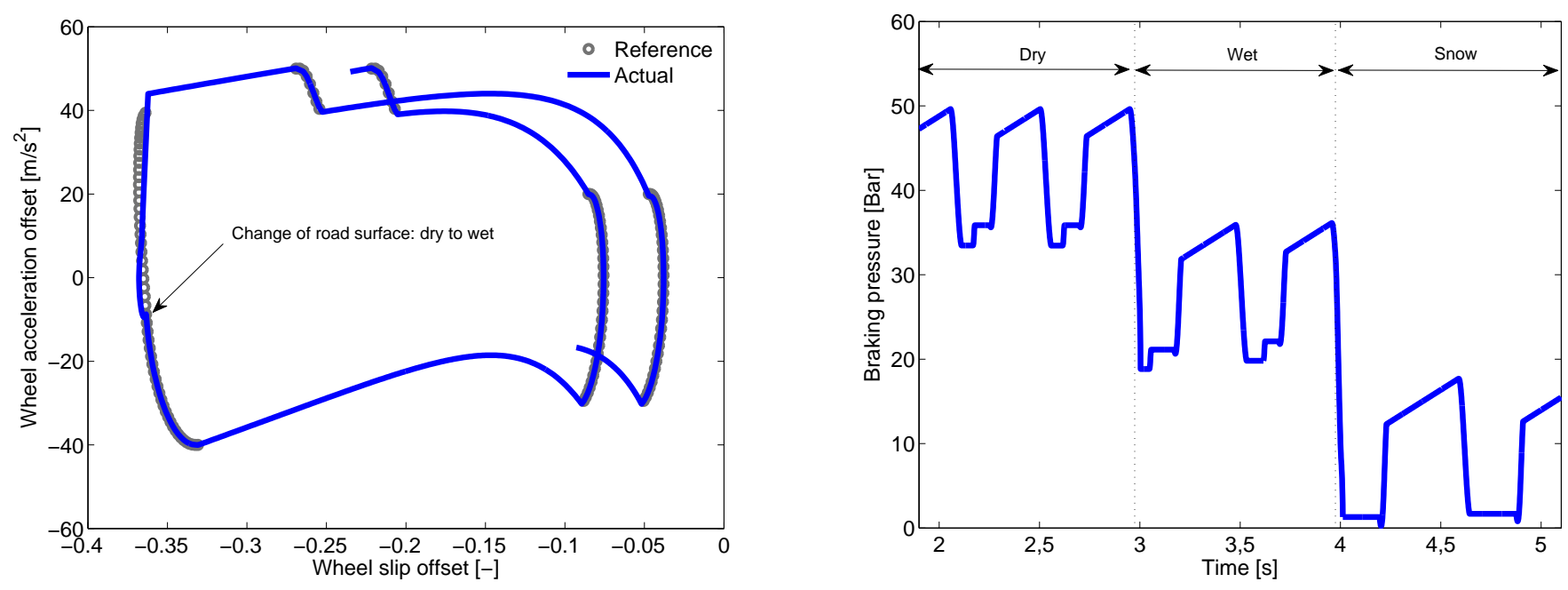

Fig. 4. Wheel acceleration tracking (during phases 1, 3, and 4) is achieved using the observer of Section IV and the control design of Section V. The car runs on dry asphalt during three seconds, then on wet asphalt for one second, and finally on snow until the end of the simulation. When the road conditions change, the brake pressure is reduced and follows the available tyre force potential.

gains are chosen:

$$
\begin{aligned}
& k_{1}^{+}=\alpha_{2}+2\left(\beta_{1}+\beta_{2}\right) \\
& k_{2}^{+}=\left(-\alpha_{1}-k_{1}^{+} \alpha_{2}-\left(\beta_{1}^{2}+\beta_{2}^{2}+4 \beta_{1} \beta_{2}\right)\right) / a \\
& k_{3}^{+}=\left(-k_{1}^{+} \alpha_{1}+a k_{2}^{+} \alpha_{2}-2 \beta_{1} \beta_{2}\left(\beta_{1}+\beta_{2}\right)\right) / a \\
& k_{4}^{+}=-\beta_{1}^{2} \beta_{2}^{2} / a .
\end{aligned}
$$

And, for negative $z_{1}$ 's,

$$
\begin{aligned}
& k_{1}^{-}=\alpha_{2}-2\left(\beta_{1}+\beta_{2}\right) \\
& k_{2}^{-}=\left(-\alpha_{1}-k_{1}^{-} \alpha_{2}-\left(\beta_{1}^{2}+\beta_{2}^{2}+4 \beta_{1} \beta_{2}\right)\right) / a \\
& k_{3}^{-}=\left(-k_{1}^{-} \alpha_{1}+a k_{2}^{-} \alpha_{2}+2 \beta_{1} \beta_{2}\left(\beta_{1}+\beta_{2}\right)\right) / a \\
& k_{4}^{-}=-\beta_{1}^{2} \beta_{2}^{2} / a,
\end{aligned}
$$

are positive constants that assign the spectrum of the error's dynamics, which has two double real eigenvalues $-\beta_{1}$ and $-\beta_{2}$.

Figure 3 shows the obtained simulation results. The details of the braking scenario are given in the figure's caption. The observer estimates accurately the values of the XBS, for different road conditions. During transitions, (which last $25 \mathrm{~ms}$ ), the estimated XBS values change abruptly. The observer cannot give good estimations during these transitions. Nevertheless, as soon as they are over, the observer error decreases in a relatively short period of time that, of course, depends on the choice of $\beta_{1}$ and $\beta_{2}$.

\section{Control Design}

A five-phase hybrid ABS algorithm [27] is described in Figure 5. Each of the algorithm's phases defines either a constant or quickly changed brake pressure $P_{b}(t)$ that is applied to the brake. The switches between each phase are triggered when the value of the wheel acceleration offset $x_{2}$ crosses some predefined threshold. The main interest of such hybrid approaches is that they do not use any information on the unmeasured variable $x_{1}$. Nevertheless, they are able to keep the wheel slip in a small neighborhood of its optimal value $\lambda^{*}$, for which the longitudinal tyre force is maximal (with the aim of minimizing the braking distance), without using explicitly the value of the optimal setpoint. The reader can find in [27] more details about this five-phase hybrid ABS algorithm.

When the algorithm of Figure 5 is tested on an experimental setup [11], it might fail to cycle correctly as soon as there are considerable changes in the environment. The main reason behind this lack of robustness is that, during the different phases, the wheel acceleration is controlled in open-loop, with a brake pressure increase that is independent of the wheel's acceleration. This shortcoming can be overcome [11] by controlling the wheel acceleration $x_{2}$ in closed-loop (around a predefined trajectory $x_{2}^{*}$ ), during the phases for which the brake torque changes quickly.

In order to do this, define $\tau:=t-t_{0}$, where $t_{0}$ is the instant at which a given phase begins. Consider the time $T$ needed by the reference trajectory $x_{2}^{*}$ to go from the previous threshold $\epsilon_{i}$ to the next one $\epsilon_{j}$. Ideally, the duration $T$ should be as small as possible but, due to the physical limitations of the brake actuator, there exists a lower bound on the achievable $T$ 's. If $\dot{P}_{b}^{M}$ is defined as the maximum brake pressure derivative that the actuator can deliver (in absolute value), then the choice of the reference trajectory $x_{2}^{*}$ must guarantee that $|\dot{P}| \leq \dot{P}_{b}^{M}$. Furthermore, in order to minimize the system's sensitivity to actuator delays, it is natural to require a zero derivative for $x_{2}^{*}$ at the beginning and at the end of each phase [11]. A possible choice for a reference trajectory $x_{2}^{*}$ that goes from $\epsilon_{i}$ to $\epsilon_{j}$ is therefore

$$
x_{2}^{*}(\tau)=a_{0}+a_{1} \tau+a_{2} \tau^{2}+a_{3} \tau^{3},
$$

where $a_{0}=\epsilon_{i}, a_{1}=0, a_{2}=-3\left(\epsilon_{i}-\epsilon_{j}\right) / T^{2}$, and $a_{3}=$ $2\left(\epsilon_{i}-\epsilon_{j}\right) / T^{3}$. By imposing, additionally, the constraint $T \geq$ $(3 / 2 b)\left|\epsilon_{i}-\epsilon_{j}\right| / \dot{P}_{b}^{M}$, one can ensure that the reference trajectory respects the brake actuator's limitations described above. 
Now, define the tracking error $\xi=x_{2}-x_{2}^{*}$ and the control law

$$
u(t)=\frac{1}{b}\left(\frac{a}{v_{x}} \hat{\mu}^{\prime}(t) x_{2}+\frac{d x_{2}^{*}(t)}{d t}-\alpha \xi(t)\right),
$$

where $\alpha>0$ is the control gain and $\hat{\mu}^{\prime}(t)$ is an estimation of the extended braking stiffness $\mu^{\prime}\left(x_{1}(t)\right)$. In the absence of estimation error, the tracking error converges exponentially to zero, provided that the control gain $\alpha$ is taken big enough. Observe, however, that the gain $\alpha$ is limited by the delay margin of the system [15]. In this approach, the choice of controlling only the variable $x_{2}$ might be surprising. But it appears that the stability of all other variables actually comes from the fact that they are bounded functions of the wheel slip $x_{1}$, which remains bounded both for hybrid [11] and continuous [28] control designs, provided that the wheel acceleration offset $x_{2}$ follows its reference.

In the simulation of Figure 4, the control uses the XBS estimation given by the observer. Thanks to the observer performance, the control law (22) ensures a good tracking performance of the wheel acceleration $x_{2}$ to its pre-defined reference. As a consequence of the robustness added by the closed-loop wheel acceleration control, the brake pressure is automatically increased or decreased to match road conditions.

\section{CONCLUSION}

In the context of anti-lock brake systems (ABS), this paper presented a new approach to estimate the extended braking stiffness. The first contribution of this work is a new nonlinear wheel acceleration model in which the XBS enters as one of the state variables. This model is obtained using either Burckhardt's model or its linearly parametrized approximation. The second contribution is the design of two stable XBS observers. When the road conditions are known, a threedimensional observer solves the problem. But, for unknown road conditions, a more complex four-dimensional observer should be used instead. In both cases, the stability of the observers is proved via time-rescaling and LaSalle-like theorems for linear switched systems.

The three-dimensional observer has been tested on experimental data coming from TU-Delft's test-rig [11]. In such tests, the parameters associated to the mounted tyre are known. The experimental results show the effectiveness of this observer. The four-dimensional observer has been tested in simulations in a scenario that includes unkown changes of road conditions. The simulation results show a precise estimation of the XBS even in the case of discontinuous jumps of road conditions.

The proposed method has nevertheless several limitations. First, it needs a (rough) estimation of the vehicle's speed (see, e.g., [10] and [9] for works that consider this problem). Second, the combined convergence of the observer and of the control law has not been proved. One could expect, however, that such a proof is obtainable via cascaded design arguments [23]. Third, the vertical load $F_{z}$ has been considered to be both known and constant. It is true that $F_{z}$ can be "reconstructed" using the longitudinal and lateral accelerations as inputs. It is also known that hybrid ABS strategies have a

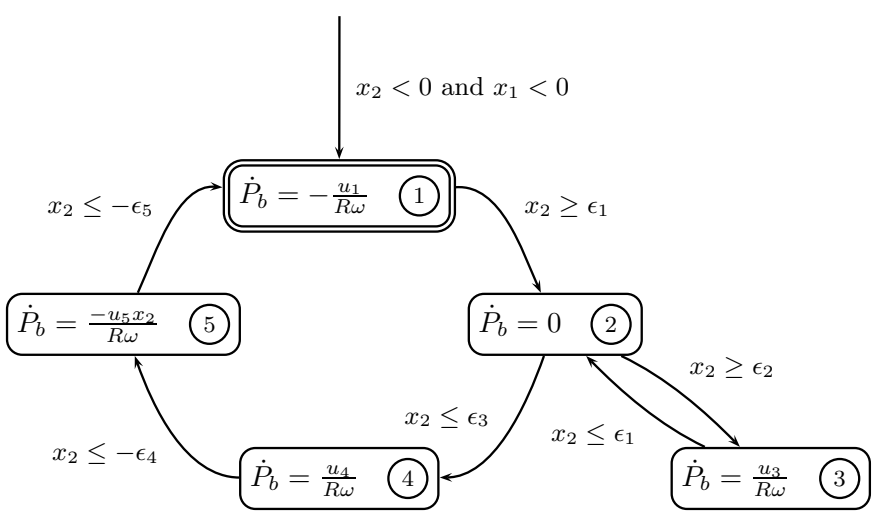

Fig. 5. The academic five-phase hybrid ABS strategy proposed in [27]. The wheel acceleration thresholds $\epsilon_{i}$ and the brake pressure increase and decrease rates $u_{i}$ must be tuned in order to obtain an asymptotically stable limit cycle (see, e.g., [1] and [27]).

certain degree of robustness with respect to vertical load uncertainties (see, e.g., the Appendix A.1 of [11]). Nevertheless, the impact of a time-varying vertical load on the proposed design is clearly a topic that deserves further investigations.

\section{APPENDIX}

This section includes a sketch of the proof of Theorem 1 . The interested reader can find in [16] an approach that generalizes this method to a more general class of switched systems that contains, as particular cases, the three and fourdimensional observers proposed in this paper.

The characteristic polynomial of the matrix $A_{+}$is given by

$$
\eta^{3}+\left(k_{1}^{+}-c\right) \eta^{2}-\left(c k_{1}^{+}+a k_{2}^{+}\right) \eta-a k_{3}^{+}=0 .
$$

Using the Routh criterion for (23) leads directly to condition (15). The same argument, but applied to $A_{-}$, gives condition (16).

Assume that the observer gains $K^{+}$and $K^{-}$satisfy, respectively, the conditions (15) and (16). For additional details concerning the following steps, the reader is referred to [13, Theorem 4]. The objective is to show that there exists a pair $\left\{P_{+}, P_{-}\right\}$, of symmetric positive definite matrices satisfying all the conditions required by that theorem, for an appropriately defined pair of matrices $\left\{C_{+}, C_{-}\right\}$.

Define $C_{+}=\left(\begin{array}{lll}c_{1}^{+} & 0 & 0\end{array}\right)$ and $C_{-}=\left(\begin{array}{lll}c_{1}^{-} & 0 & 0\end{array}\right)$, where $c_{1}^{+}, c_{1}^{-} \neq 0$. It is easy to check that the pairs $\left(A_{+}, C_{+}\right)$ and $\left(A_{-}, C_{-}\right)$are observable. In order to satisfy the conditions of [13, Theorem 4], one must find a matrix $P$ that satisfies simultaneously the equations $A_{+}^{T} P+P A_{+}=-C_{+}^{T} C_{+}$and $A_{-}^{T} P+P A_{-}=-C_{-}^{T} C_{-}$. Observe that $P$ defines a nonstrict Lyapunov function only, because the symmetric matrices $C_{i}^{T} C_{i}$ are not definite positive. Denote by $\left(p_{i j}\right)$ the elements of $P$. One can easily deduce from $A_{+}^{T} P+P A_{+}=$ $-C_{+}^{T} C_{+}$that

$$
\begin{array}{ll}
p_{11}=\frac{c^{2}-\left(c k_{1}^{+}+a k_{2}^{+}\right)}{a^{2}} p_{22}, & p_{12}=\frac{c}{a} p_{22}, \\
p_{13}=\frac{1}{a} p_{22}, \quad p_{23}=0, \quad \text { and } \quad p_{33}=\frac{\left(c-k_{1}^{+}\right)}{a k_{3}^{+}} p_{22},
\end{array}
$$


where $p_{22}>0$. With the elements of $P$ computed as in (24), one obtains

$$
c_{1}^{+}= \pm \sqrt{2 \frac{\left(c-k_{1}^{+}\right)\left(c k_{1}^{+}+a k_{2}^{+}\right)+a k_{3}^{+}}{a^{2}} p_{22}} \neq 0 .
$$

The term in the square root is positive because of (15) and $p_{22}>0$.

Similarly, since $P$ has to satisfy the condition $A_{-}^{T} P+$ $P A_{-}=-C_{-}^{T} C_{-}$, it follows that the elements of $P$ are also of the form

$$
\begin{aligned}
& p_{11}=\frac{c^{2}-\left(c k_{1}^{-}+a k_{2}^{-}\right)}{a^{2}} p_{22}, \quad p_{12}=\frac{c}{a} p_{22}, \\
& p_{13}=\frac{1}{a} p_{22}, \quad p_{23}=0, \quad \text { and } \quad p_{33}=\frac{\left(c-k_{1}^{-}\right)}{a k_{3}^{-}} p_{22} .
\end{aligned}
$$

From (24) and (26), additional conditions on the observer gains $K^{+}$and $K^{-}$can be obtained:

$$
\begin{aligned}
\frac{(c-k-1)}{a k_{3}^{-}}= & \frac{\left(c-k_{1}^{+}\right)}{a k_{3}^{+}}>0 \text { and } \\
& \left(c k_{1}^{+}+a k_{2}^{+}\right)=\left(c k_{1}^{-}+a k_{2}^{-}\right)<0 .
\end{aligned}
$$

The element $c_{1}^{-}$of $C_{-}$is also different from zero and, because of (16) and $p_{22}>0$, one obtains

$$
c_{1}^{-}= \pm \sqrt{-2 \frac{\left(c-k_{1}^{-}\right)\left(c k_{1}^{-}+a k_{2}^{-}\right)+a k_{3}^{-}}{a^{2}} p_{22}},
$$

which ends the proof.

\section{REFERENCES}

[1] I. Ait-Hammouda and W. Pasillas-Lépine. Jumps and synchronization in anti-lock brake algorithms. In Proceedings of the Advanced Vehicle Control Congress, Kobe (Japan), 2008.

[2] D. Angeli. A note on stability of arbitrarily switched homogeneous systems. Nonlinear Control Abstracts, 1(13), 1999.

[3] G. Besançon. Remarks on nonlinear adaptive observer design. Systems \& Control Letters, 41(4):271-280, 2000.

[4] R. Bosch Gmbh. Automotive Handbook. Bentley, 2004.

[5] M. Burckhardt. Fahrwerktechnik: Radschlupf-Regelsysteme. VogelVerlag, 1993.

[6] R.D. Castro, R.E. Araujo, and D. Freitas. Real-time estimation of tyreroad friction peak with optimal linear parameterisation. IET Control Theory \& Applications, 6(14):2257-2268, 2012.

[7] S.B. Choi. Antilock brake system with a continuous wheel slip control to maximize the braking performance and the ride quality. IEEE Transactions on Control Systems Technology, 16(5):996-1003, 2008.

[8] M. Corno, M. Gerard, M. Verhaegen, and E. Holweg. Hybrid ABS control using force measurement. IEEE Transactions on Control Systems Technology, 20(5):1223-1235, 2012.

[9] M. Corno, G. Panzani, and S. M. Savaresi. Traction-control-oriented state estimation for motorcycles. IEEE Transactions on Control Systems Technology, 21(6):2400-2407, 2013.

[10] A. Daiss and U. Kiencke. Estimation of vehicle speed: Fuzzy-estimation in comparison with Kalman-filtering. In Proceedings of the Conference on Control Applications, pages 281-284, 1995.

[11] M. Gerard, W. Pasillas-Lépine, E. De Vries, and M. Verhaegen. Improvements to a five-phase ABS algorithm for experimental validation. Vehicle System Dynamics, 50(10):1585-1611, 2012.

[12] F. Gustafsson. Monitoring tire-road friction using the wheel slip. Control Systems, IEEE, 18(4):42-49, 1998.

[13] J.P. Hespanha. Uniform stability of switched linear systems: Extensions of LaSalle's invariance principle. IEEE Transactions on Automatic Control, 49(4):470-482, 2004.

[14] F.B. Hildebrand. Introduction to numerical analysis - Second Edition. Dover Publications, 1974
[15] T.B. Hoang, W. Pasillas-Lépine, and A. De Bernardinis. Reducing the impact of wheel-frequency oscillations in continuous and hybrid ABS strategies. In Proceedings of the Advanced Vehicle Control Congress, Seoul (Korea), 2012.

[16] T.B. Hoang, W. Pasillas-Lépine, and W. Respondek. A switching observer for systems with linearizable error dynamics via singular timescaling. In Mathematical Theory of Networks and Systems, 2014. Submitted for publication.

[17] T.A. Johansen, I. Petersen, J. Kalkkuhl, and J. Ludemann. Gainscheduled wheel slip control in automotive brake systems. IEEE Transactions on Control Systems Technology, 11(6):799-811, 2003.

[18] U. Kiencke and L. Nielsen. Automotive Control Systems. SpringerVerlag New York, Inc., 2000.

[19] C. Y. Kuo and E. C. Yeh. A four-phase control scheme of an antiskid brake system for all road conditions. IMechE Part D: Journal of Automobile Engineering, 206(4):275-283, 1992.

[20] H. Leiber and A. Czinczel. Antiskid system for passenger cars with a digital electronic control unit. SAE paper, (790458), 1979.

[21] D. Liberzon. Switching in Systems and Control. Springer, 2003.

[22] D. Liberzon and A.S. Morse. Basic problems in stability and design of switched systems. IEEE Control Systems, 19(5):59-70, 1999.

[23] A. Loría and E. Panteley. Cascaded nonlinear time-varying systems: Analysis and design. In F. Lamnabhi-Lagarrigue, A. Loría, and E. Panteley, editors, Advanced Topics in Control Systems Theory, pages 579579. Springer, 2005.

[24] B.J. Olson, S.W. Shaw, and G. Stépán. Nonlinear dynamics of vehicle traction. Vehicle System Dynamics, 40(6):377-399, 2003.

[25] E. Ono, K. Asano, M. Sugai, S. Ito, M. Yamamoto, M. Sawada, and Y. Yasui. Estimation of automotive tire force characteristics using wheel velocity. Control Engineering Practice, 11(12):1361-1370, 2003.

[26] H. Pacejka. Tyre and Vehicle Dynamics. Butterworth-Heinemann, 2005.

[27] W. Pasillas-Lépine. Hybrid modeling and limit cycle analysis for a class of five-phase anti-lock brake algorithms. Vehicle System Dynamics, 44(2):173-188, 2006.

[28] W. Pasillas-Lépine, A. Loría, and M. Gerard. Design and experimental validation of a nonlinear wheel slip control algorithm. Automatica, 48(8):1852-1859, 2012

[29] R. Prony. Essai experimental et analytique: sur les lois de la dilatabilite de fluides [...]. J. de l'Ecole Polytechnique (Paris), 1(2):24-76, 1795.

[30] S. Savaresi, M. Tanelli, and C. Cantoni. Mixed slip-deceleration control in automotive braking systems. Journal of Dynamic Systems, Measurement, and Control, 129(1):20-31, 2007.

[31] Z. Shida, R. Sakurai, M. Watanabe, Y. Kano, and M. Abe. A study on effects of tire characteristics on stop distance of ABS braking with simplified model. In Proceedings of the Advanced Vehicle Control Congress, Loughborough, UK, 2010

[32] M. Sugai, H. Yamaguchi, M. Miyashita, T. Umeno, and K. Asano. New control technique for maximizing braking force on antilock braking system. Vehicle System Dynamics, 32(4-5):299-312, 1999.

[33] M. Tanelli, L. Piroddi, and S.M. Savaresi. Real-time identification of tire-road friction conditions. IET Control Theory \& Applications, 3(7):891-906, 2009.

[34] C. Unsal and P. Kachroo. Sliding mode measurement feedback control for antilock braking systems. IEEE Transactions on Control Systems Technology, 7(2):271-281, 1999.

[35] A. van Zanten. Evolution of electronic control systems for improving the vehicle dynamic behavior. In Proceedings of the Advanced Vehicle Control Congress, Tokyo, Japan, 2002.

[36] J. Villagra, B. d'Andréa Novel, M. Fliess, and H. Mounier. A diagnosisbased approach for tire-road forces and maximum friction estimation. Control Engineering Practice, 19(2):174-184, 2011.

[37] P.W.A. Zegelaar. The dynamic response of tyres to brake torque variations and road unevennesses. Delft University of Technology, 1998. 